\title{
Endovascular Coiling versus Surgical Clipping for Poor-Grade Ruptured Intracranial Aneurysms: Postoperative Complications and Clinical Outcome in a Multicenter Poor-Grade Aneurysm Study
}

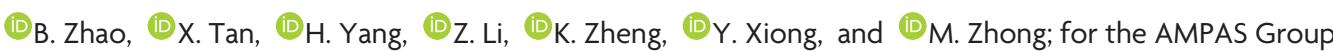

\begin{abstract}
BACKGROUND AND PURPOSE: Endovascular coiling is an alternative to surgical clipping for ruptured intracranial aneurysms. However, no large multicenter prospective study has compared coiling and clipping in patients with poor-grade ruptured aneurysms. We aimed to determine differences in postoperative complications and clinical outcome between the 2 treatments in this group of patients.
\end{abstract}

MATERIALS AND METHODS: A Multicenter Poor-Grade Aneurysm Study was a prospective, multicenter, observational registry of consecutive patients who presented with poor-grade ruptured aneurysms. Three hundred sixty-six patients were enrolled from October 2010 to March 2012. "Poor-grade aneurysm" was defined as a World Federation of Neurosurgical Societies grade of IV or V at the time of aneurysm treatment. Two hundred sixty-two patients received aneurysm treatment within 21 days and were included. Clinical outcomes were assessed at discharge and at 6 and 12 months by the modified Rankin Scale.

RESULTS: One hundred thirty-three (50.8\%) patients underwent endovascular coiling. Unadjusted analysis showed that the outcome rate (mRS $0-1$ or mRS $0-2$ ) at 6 and 12 months in patients undergoing coiling was higher than that in patients undergoing clipping. In adjusted analyses, there was no statistically significant difference in outcomes at 6 and 12 months between the 2 groups. The risk of radiologic hydrocephalus was higher in patients treated with coiling than that in those treated with clipping (adjusted OR, 3.36; 95\% $\mathrm{Cl}$, 1.13-10.01; $P=.030$ ).

CONCLUSIONS: The long-term outcome in selected patients was similar between endovascular coiling and clipping for poor-grade ruptured aneurysms. The risk of radiologic hydrocephalus was higher after endovascular treatment of poor-grade aneurysms.

ABBREVIATIONS: AMPAS = A Multicenter Poor-Grade Aneurysm Study; aSAH = aneurysmal subarachnoid hemorrhage; EVD = external ventricular drainage; GCS = Glasgow Coma Scale; WFNS = World Federation of Neurosurgical Societies; ISAT = International Subarachnoid Aneurysm Trial

ntracranial aneurysm rupture leading to aneurysmal subarachnoid hemorrhage $(\mathrm{aSAH})$ is a devastating condition. Poorgrade ruptured aneurysms account for approximately $30 \%$ of all cases of aSAH. ${ }^{1,2}$ Traditionally, patients with poor-grade aneurysms have been managed medically. In the past 2 decades, aggressive treatment has been proposed to improve outcomes in selected patients. ${ }^{1,3,4}$ Despite recent advances in the treatment of

Received September 22, 2015; accepted after revision November 8.

From the Department of Neurosurgery (B.Z., X.T., Z.L., K.Z., Y.X., M.Z.), The First Affiliated Hospital of Wenzhou Medical University, Wenzhou, China; Department of Neurosurgery (B.Z.), Mayo Clinic, Rochester, Minnesota; and Department of Neurosurgery (H.Y.), The First Affiliated Hospital of Guizhou Medical University, Guiyang, China.

This work was supported by the Chinese Ministry of Health (grant WKJ2010-2-016), the Ministry of Science and Technology of China (grant 2011BAI08B06), and Wenzhou Bureau of Science and Technology (grant Y20090005). The funding organizations were not involved in the design and conduct of the study.

Please address correspondence to Ming Zhong, MD, Department of Neurosurgery, The First Affiliated Hospital of Wenzhou Medical University, Nanbai Xiang Town, 325000 Wenzhou, China; e-mail: zhongming158@sohu.com

-- Indicates open access to non-subscribers at www.ajnr.org

http://dx.doi.org/10.3174/ajnr.A4649
aSAH, patients with poor-grade aneurysms have high rates of long-term morbidity and mortality. ${ }^{1,3-6}$

The International Subarachnoid Aneurysm Trial (ISAT) has demonstrated that for ruptured aneurysms suitable for endovascular embolization and clipping, patients treated with coiling had better outcomes than those treated with clipping. ${ }^{7,8}$ However, only a few patients $(4.4 \%)$ in ISAT had poor-grade aneurysms. The results may not directly apply to poor-grade patients. Currently, case series have shown that endovascular coiling is a viable alternative to clipping for poor-grade aneurysms. ${ }^{5,9,10}$ However, to date, no randomized, controlled study has been performed to evaluate the efficacy of coiling versus clipping for poor-grade ruptured aneurysms, to our knowledge. There has been no large multicenter prospective study regarding the effects of different treatments on postoperative complications and clinical outcome in these patients.

A Multicenter Poor-Grade Aneurysm Study (AMPAS) was a prospective, multicenter, observational registry of consecutive patients who presented with poor-grade ruptured aneurysm. ${ }^{11}$ The primary purpose of this report was to determine differences 


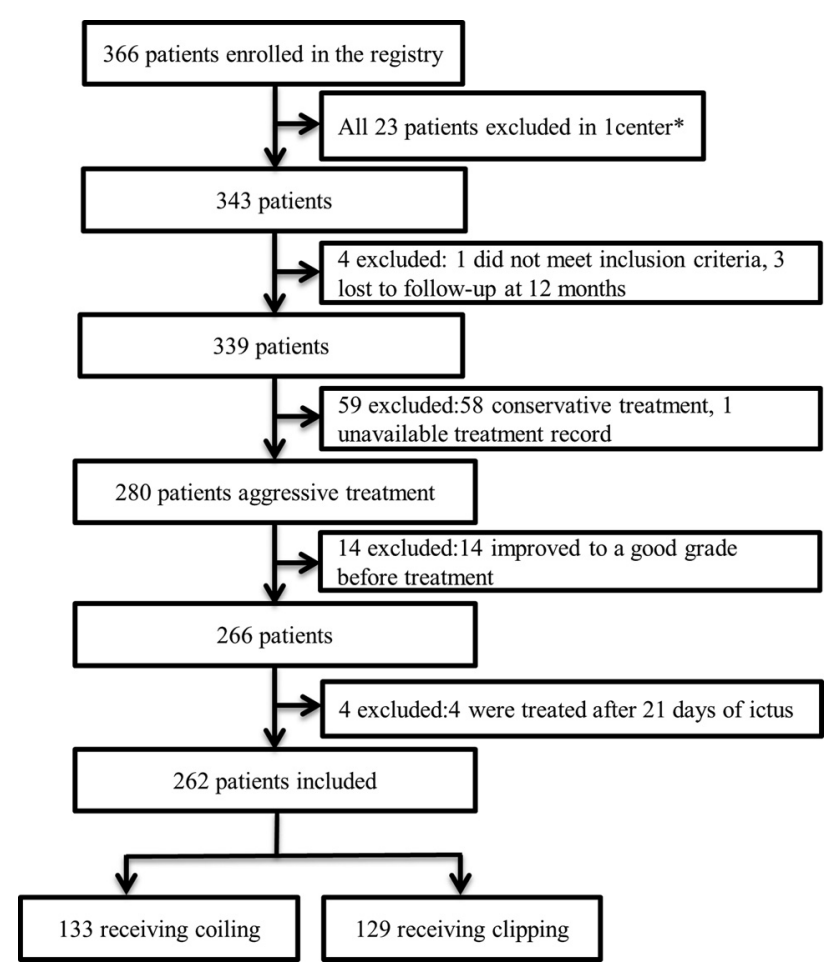

FIG 1. Study flow diagram. The asterisk indicates that 1 center was excluded because enrollment was not consecutive and 8 patients were lost to follow-up.

in postoperative complications and clinical outcome between patients treated with coiling and those treated with clipping in the AMPAS after adjustment for differences in baseline confounders.

\section{MATERIALS AND METHODS Study Design}

The AMPAS protocol was approved by the Chinese Ethics Committee of Registering Clinical Trials (ChiECRCT-2010019) and was published elsewhere. ${ }^{11}$ The study was registered with the Chinese Clinical Trail Registry (No. ChiCTR-TNRC-10001041). Between October 2010 and March 2012, 366 patients from 11 centers were enrolled in the registry. All informed consent was obtained from the patients' legal representatives. All patients were treated at tertiary referral centers (with $>150$ aSAH cases per year) with expertise in aneurysm coiling and clipping. "Poorgrade aneurysm" was defined as a World Federation of Neurosurgical Societies (WFNS) grade of IV or V after aSAH. Patients between 18 and 75 years of age were eligible for entry if they presented with poor-grade subarachnoid hemorrhage and had a ruptured aneurysm as confirmed by CT angiography or digital subtraction angiography. Patients were not enrolled if their family refused any treatment and they died in the emergency department. In this report, patients were excluded if they improved from an initial poor grade to a good grade (WFNS grade I or II or III) before aneurysm treatment and if they underwent aneurysm treatment after 21 days of poor-grade aSAH. Of the 366 patients, 262 patients were included in this study (Fig 1).

\section{Clinical Treatment Protocol}

The treatment protocol included aggressive resuscitation, intensive critical care, early CT angiography or cerebral angiography if the patient was clinically stabilized, multidisciplinary consensus, and early treatment. ${ }^{11-13}$ The multidisciplinary team consisting of vascular neurosurgeons, interventional neuroradiologists, and anesthesiologists, made the treatment decision by considering patients' clinical conditions and radiologic findings. Surgical treatment was regularly performed in patients with associated large intracerebral hemorrhage $(>30 \mathrm{~mL})$, and endovascular coiling was performed in patients with posterior circulation aneurysms. Following aneurysm treatment, all patients were transferred to a neurointensive care unit and underwent aggressive management for vasospasm according to the individual institutional standards of practice.

\section{Data Collection and Definition}

The data entry form included information on demographic characteristics, medical history, clinical presentation, neurologic examination, radiologic findings at the time of treatment, aneurysm characteristics, type and timing of treatment, postoperative complications during hospitalization, and outcomes at discharge and at 6 and 12 months. The Glasgow Coma Scale (GCS) and WFNS grade were the last assessment obtained before sedation or intubation if the patient was sedated or intubated. The Fisher grade and modified Fisher grade were determined by the last CT scan before treatment. The time of treatment was defined as the interval between the time of the poor-grade condition after ictus and the time of aneurysm treatment.

\section{Outcomes Measures}

The outcome was assessed at each center by an independent neurosurgeon by using the modified Rankin Scale. They were not involved in the treatment of patients. The primary outcome measure was functional outcome at discharge and 6 and 12 months. The secondary outcomes were postoperative complications. Major complications included aneurysm rebleeding, cerebral infarction, symptomatic vasospasm, radiologic and clinical hydrocephalus, seizure, pneumonia, and renal failure. "Radiologic hydrocephalus" was defined as ventricular dilation with enlarged temporal horns ( $\geq 2 \mathrm{~mm}$ wide) on postoperative CT, ${ }^{14,15}$ and "clinical hydrocephalus" was defined as the presence of postoperative external ventricular drainage (EVD), ventriculoperitoneal shunt or lumbar drain placement, and the presence of radiologic hydrocephalus. "Cerebral infarction" was defined as a new hypoattenuation on posttreatment CT, and "symptomatic vasospasm" was defined as neurologic deterioration not explained by other causes such as seizure, hydrocephalus, edema, and a new hemorrhage.

\section{Statistical Analyses}

Data were analyzed with SPSS, Version 22.0 (IBM, Armonk, New York). Differences in baseline characteristics were compared between the coiling and clipping groups. An independent-samples $t$ test and Mantel-Haenszel, $\chi^{2}$, and Fisher exact tests were used to find variations in patient characteristics between the 2 groups. Crude and adjusted odds ratios with 95\% confidence intervals were calculated with logistic regression analysis for major complications and outcomes ( $\mathrm{mRS} 0-1, \mathrm{mRS} 0-2$, mRS $0-3$, and death) at discharge and at 6 and 12 months for the different treatment 
groups. All analyses were adjusted for smoking history, pretreatment GCS score, WFNS grade, Fisher grade, modified Fisher grade, brain herniation, ruptured aneurysm location, and postoperative EVD. In univariate analysis of the association between radiologic and clinical hydrocephalus and clinical characteristics, clinical variables with a $P$ value $\leq .05$ were entered into the multivariate analysis model by using the backward logistic regression method to identify independent risk factors for hydrocephalus. Receiver operating characteristic of area under the curve analysis was performed to test the prediction ability of the model. A $P$ value $<.05$ was considered statistically significant.

\section{RESULTS}

\section{Patient Characteristics}

Baseline characteristics of the 262 patients are shown in Table 1. According to multidisciplinary consensus, 133 (50.8\%) patients received endovascular coiling within 21 days after poor-grade aSAH. Patients treated with coiling more often had a history of smoking $(P=.035)$, a higher GCS score $(P<.001)$, a WFNS grade of IV $(P=.002)$, a lower Fisher grade $(P<.001)$ and modified

Table 1: Baseline characteristics

\begin{tabular}{lccr}
\hline \multicolumn{1}{c}{ Variables } & Coiling $(\boldsymbol{n}=133)$ & Clipping $(\boldsymbol{n}=129)$ & $\boldsymbol{P}$ Value \\
\hline Age (yr) & $54.5(11.8)$ & $54.4(10.9)$ & .960 \\
Women & $62(46.6 \%)$ & $69(53.5 \%)$ & .266 \\
Hypertension & $50(37.6 \%)$ & $63(48.8 \%)$ & .066 \\
Current smoking & $49(36.8 \%)$ & $32(24.8 \%)$ & .035 \\
Diabetes mellitus & $10(7.5 \%)$ & $4(3.1 \%)$ & .168 \\
Previous ischemic stroke & $3(2.3 \%)$ & $4(3.1 \%)$ & .719 \\
Previous SAH & $4(3.0 \%)$ & $9(7.0 \%)$ & .163 \\
GCS score & $7.5(2.6)$ & $6.4(2.4)$ & $<.001$ \\
WFNS grade & & & .002 \\
Grade IV & $82(61.7 \%)$ & $55(42.6 \%)$ & \\
Grade V & $51(38.3 \%)$ & $74(57.4 \%)$ & \\
Fisher grade & $3.1(0.9)$ & $3.6(0.7)$ & $<.001$ \\
Modified Fisher grade & $2.5(1.1)$ & $2.9(0.9)$ & .001 \\
Intraventricular hemorrhage & $52(39.1 \%)$ & $51(38.8 \%)$ & .955 \\
Brain herniation & $17(12.8 \%)$ & $51(39.5 \%)$ & $<.001$ \\
Multiple aneurysms & $22(16.5 \%)$ & $24(18.6 \%)$ & .661 \\
Aneurysm size (mm) & $5.7(2.9)$ & $6.0(3.9)$ & .536 \\
Ruptured aneurysm location & & & $<.001$ \\
Anterior circulation & $107(80.5 \%)$ & $128(99.2 \%)$ & \\
Posterior circulation & $26(19.5 \%)$ & $1(0.8 \%)$ & \\
Preoperative EVD & $10(7.5 \%)$ & $11(8.5 \%)$ & .778 \\
Postoperative EVD & $37(27.8 \%)$ & $8(6.2 \%)$ & $<.001$ \\
Time to treatment & & & .544 \\
0-72 hr & $101(75.9 \%)$ & $102(79.1 \%)$ & \\
73 hr to 21 days & $32(24.1 \%)$ & $27(21.1 \%)$ & .721 \\
Hospital stay (days) & $24.3(20.7)$ & $23.3(21.4)$ & \\
\hline Data a & & & \\
\hline
\end{tabular}

${ }^{a}$ Data are mean (SD) or $n / N(\%)$.

Table 2: Major complications associated with coiling compared with clipping

\begin{tabular}{|c|c|c|c|c|c|c|}
\hline \multirow[b]{2}{*}{ Complications } & \multirow[b]{2}{*}{ Coiling } & \multirow[b]{2}{*}{ Clipping } & \multicolumn{2}{|c|}{ Crude Analysis } & \multicolumn{2}{|c|}{ Adjusted Analysis } \\
\hline & & & OR $(95 \% \mathrm{CI})$ & $P$ Value & OR $(95 \% \mathrm{Cl})$ & $P$ Value \\
\hline Aneurysm rebleeding & $6(4.5 \%)$ & $2(1.6 \%)$ & $3.00(0.59-15.15)$ & .180 & $3.41(0.55-21.27)$ & .189 \\
\hline Cerebral infarction & 15 (11.3\%) & $23(17.8 \%)$ & $0.59(0.29-1.18)$ & .140 & $0.62(0.26-1.49)$ & .283 \\
\hline Symptomatic vasospasm & $14(10.5 \%)$ & $13(10.1 \%)$ & $1.05(0.47-2.33)$ & .910 & $0.53(0.18-1.59)$ & .259 \\
\hline Radiologic hydrocephalus & 19 (14.3\%) & $7(5.4 \%)$ & $2.91(1.18-7.17)$ & .020 & 3.36 (1.13-10.01) & .030 \\
\hline Clinical hydrocephalus & 14 (10.5\%) & $2(1.6 \%)$ & $7.47(1.66-33.57)$ & .009 & $5.15(0.95-27.89)$ & .057 \\
\hline Seizure & $5(3.8 \%)$ & $6(4.7 \%)$ & $0.80(0.24-2.69)$ & .720 & $0.72(0.18-2.85)$ & .641 \\
\hline Pneumonia & $31(23.3 \%)$ & $39(30.2 \%)$ & $0.70(0.41-1.22)$ & .210 & $0.61(0.30-1.24)$ & .173 \\
\hline Renal failure & $2(1.5 \%)$ & $5(3.9 \%)$ & $0.38(0.07-1.99)$ & .250 & $0.24(0.02-2.45)$ & .230 \\
\hline
\end{tabular}

Fisher grade $(P=.001)$, and a ruptured posterior circulation aneurysm $(P<.001)$ than those treated with clipping. Patients treated with clipping more commonly experienced brain herniation than did those treated with coiling $(P<.001)$. Postoperative EVD was more often performed in patients treated with coiling $(P<.001)$.

\section{Major Postoperative Complications}

Major complications are shown in Table 2. There were no significant differences in aneurysm rebleeding, cerebral infarction, symptomatic vasospasm, seizure, pneumonia, and renal failure between the coiling and clipping groups. Patients treated with coiling had higher risks of radiologic and clinical hydrocephalus than those treated with clipping (unadjusted OR, 2.91; $P=.021$; and unadjusted OR, 7.47; $P=.009$, respectively). The risk of radiologic hydrocephalus in the coiling group was higher than that in the clipping group (adjusted OR, 3.36; $P=.030$ ), and there was a trend toward clinical hydrocephalus in the patients with coiling (adjusted OR, 5.15; $P=.057$ ) when adjusting for the status of smoking, GCS score, WFNS grade, Fisher grade, modified Fisher grade, ruptured aneurysm location, brain herniation, and postoperative EVD (Table 2).

Univariate analysis of the association between hydrocephalus and clinical characteristics showed that higher modified Fisher grade $(P=.019)$, postoperative $\operatorname{EVD}(P=.004)$, and endovascular treatment $(P=.021)$ were associated with radiologic hydrocephalus. Multivariate analysis showed that higher modified Fisher grade $(P=.012)$ and endovascular coiling $(P=.035)$ were independent risk factors for hydrocephalus (Table 3 ). The 2 variables predicted the risk of radiologic hydrocephalus with an area under the curve of 0.71 (95\% CI, 0.60-0.81; P = .001). The multivariate analysis for clinical hydrocephalus showed that postoperative $\operatorname{EVD}(P=.001)$ was an independent predictor of clinical hydrocephalus (Table 3). The model combining coiling technique and postoperative EVD predicted clinical hydrocephalus with an area under the curve of 0.79 (95\% CI, $0.66-0.91 ; P<.001)$. 


\section{Outcome at Discharge}

At discharge, $52(19.8 \%)$ patients had an mRS score of 0 or 1,78 (29.8\%) had an mRS score of $0-2,85$ (32.4\%) had an mRS score of $0-3$, and 51 (19.5\%) had died. Unadjusted analysis showed that the rate of outcome (mRS $0-2$ or $\mathrm{mRS} 0-3$ ) at discharge in the patients with coiling was higher than that in the patients with clipping. There was no significant difference in outcome at discharge after adjustment for the status of smoking, GCS score, WFNS grade, Fisher grade, modified Fisher grade, ruptured aneurysm location, brain herniation, and postoperative EVD (Table 4).

\section{Long-Term Outcomes}

At 12 months, $95(36.3 \%)$ patients had an mRS score of 0 or 1,115 (43.9\%) had an mRS of $0-2,126(48.1 \%)$ had an mRS of $0-3$, and $103(39.3 \%)$ had died. The outcomes at 12 months according to the WFNS grade and treatment technique are shown in Fig 2. Patients with a WFNS grade of V had worse outcomes than those with a WFNS grade of IV $(P<.001)$. Compared with outcomes at discharge, the rate of good outcome (mRS 0-2) increased to $39.3 \%$ at 6 months and $43.9 \%$ at 12 months. The outcome was improved after endovascular coiling or clipping with time (Table 4). Unadjusted analysis showed that the outcome rate (mRS $0-1$ or mRS $0-2$ ) at 6 and 12 months in patients with coiling was higher than that in the patients with clipping. However, there was no significant difference in outcomes at 6 months and 12 months between the 2 groups after adjustment for the status of smoking, GCS score, WFNS grade, Fisher grade, modified Fisher grade, ruptured aneurysm location, brain herniation, and postoperative EVD (Table 4).

Table 3: Multivariate analysis of predictors of hydrocephalus

\begin{tabular}{|c|c|c|c|c|}
\hline \multirow[b]{2}{*}{ Predictors } & \multicolumn{2}{|c|}{ Univariate Analysis } & \multicolumn{2}{|c|}{ Multivariate Analysis } \\
\hline & OR $(95 \% \mathrm{Cl})$ & $P$ Value & OR $(95 \% \mathrm{Cl})$ & $P$ Value \\
\hline \multicolumn{5}{|l|}{ Radiologic hydrocephalus } \\
\hline Fisher grade & $1.91(1.00-3.66)$ & .050 & & \\
\hline Modified Fisher grade & 1.79 (1.10-2.92) & .019 & $1.88(1.15-3.08)$ & .012 \\
\hline Endovascular coiling & $2.91(1.18-7.17)$ & .021 & $2.85(1.08-7.57)$ & .035 \\
\hline Postoperative EVD & $3.59(1.51-8.55)$ & .004 & & \\
\hline \multicolumn{5}{|l|}{ Clinical hydrocephalus } \\
\hline Endovascular coiling & $7.47(1.66-33.57)$ & .009 & $4.27(0.89-20.45)$ & .070 \\
\hline Postoperative EVD & $10.05(3.43-29.40)$ & $<.001$ & $6.79(2.22-20.71)$ & .001 \\
\hline
\end{tabular}

\section{DISCUSSION}

The AMPAS was a prospective, multicenter, observational registry of consecutive patients who presented with poor-grade ruptured aneurysm and was reflective of a real-world practice of multidisciplinary treatment of poor-grade aneurysms. Some patients experienced further clinical improvement with time. In this exploratory analysis, there was no significant difference in outcomes at 6 and 12 months between the 2 treatments. However, patients treated with coiling had a higher risk of radiologic hydrocephalus than those treated with clipping, and there was a trend toward clinical hydrocephalus in the patients with coiling.

Although numerous studies have reported outcomes in selected patients with poor-grade aneurysms, ${ }^{1,2,4,5,9,10}$ interpretation of the results from case series or from single-center retrospective studies has been hampered by the use of different clinical grading scales (WFNS grade or Hunt and Hess scale) and outcome measurement scores and heterogeneity in the type of treatment and in the timing of outcome assessments. ${ }^{11}$ In this study, standard neurologic scales, risk factor data, and functional outcome scores were prospectively collected in all sites. The registry dataset is also representative of the current practice for poorgrade aneurysms in high-volume centers. Our results are slightly better than those reported in older studies (good outcome of $38.3 \%-40.0 \%)^{1,2}$ and are in agreement with current studies showing a good outcome of approximately $50 \% .{ }^{10,16}$ These findings might suggest that there is an increase in the proportion of patients experiencing good outcomes in recent years. In addition, our results also support the recent study showing that poor-grade patients have experienced delayed recovery with time. ${ }^{16}$ Cerebral recovery may be attributable to cerebral plasticity. ${ }^{16}$

Since the ISAT study, endovascular coiling is more commonly used than clipping for ruptured aneurysms. Our results also show that most patients with a WFNS grade of IV and almost all patients with posterior circulation aneurysms received endovascular treatment. Unadjusted comparisons of outcomes showed that patients treated with coiling

Table 4: Clinical outcomes associated with coiling compared with clipping

\begin{tabular}{|c|c|c|c|c|c|c|}
\hline \multirow[b]{2}{*}{ Outcomes } & \multirow[b]{2}{*}{ Coiling } & \multirow[b]{2}{*}{ Clipping } & \multicolumn{2}{|c|}{ Crude Analysis } & \multicolumn{2}{|c|}{ Adjusted Analysis } \\
\hline & & & OR $(95 \% \mathrm{CI})$ & $P$ Value & OR $(95 \% \mathrm{Cl})$ & $P$ Value \\
\hline \multicolumn{7}{|l|}{ At discharge } \\
\hline mRS (0-1) & 30 (22.6\%) & 22 (17.1\%) & $1.42(0.77-2.62)$ & .266 & $0.62(0.28-1.38)$ & .241 \\
\hline $\mathrm{mRS}(0-2)$ & $48(36.1 \%)$ & $30(23.3 \%)$ & $1.86(1.09-3.20)$ & .024 & $0.74(0.35-1.60)$ & .448 \\
\hline $\mathrm{mRS}(0-3)$ & $51(38.3 \%)$ & $34(26.4 \%)$ & $1.74(1.03-2.94)$ & .039 & $0.66(0.31-1.39)$ & .273 \\
\hline Death & $27(20.3 \%)$ & $24(18.6 \%)$ & $1.11(0.60-2.06)$ & .729 & $1.88(0.84-4.25)$ & .127 \\
\hline \multicolumn{7}{|l|}{ At $6 \mathrm{Mo}$} \\
\hline mRS (0-1) & $51(38.3 \%)$ & $29(22.5 \%)$ & 2.15 (1.25-3.69) & .006 & $1.34(0.69-2.62)$ & .394 \\
\hline $\mathrm{mRS}(0-2)$ & $61(45.9 \%)$ & $42(32.6 \%)$ & $1.76(1.06-2.90)$ & .028 & $0.92(0.48-1.79)$ & .808 \\
\hline $\mathrm{mRS}(0-3)$ & $66(49.6 \%)$ & $54(41.9 \%)$ & $1.37(0.84-2.23)$ & .208 & $0.64(0.33-1.26)$ & .195 \\
\hline Death & $47(35.3 \%)$ & $52(40.3 \%)$ & $0.81(0.49-1.33)$ & .407 & $1.80(0.91-3.54)$ & .091 \\
\hline \multicolumn{7}{|l|}{ At $12 \mathrm{Mo}$} \\
\hline mRS (0-1) & $58(43.6 \%)$ & $37(28.7 \%)$ & $1.92(1.15-3.21)$ & .012 & 0.98 (0.51-1.91) & .961 \\
\hline $\mathrm{mRS}(0-2)$ & $68(51.1 \%)$ & $47(36.4 \%)$ & 1.83 (1.11-2.99) & .017 & $0.75(0.38-1.49)$ & .410 \\
\hline $\mathrm{mRS}(0-3)$ & $71(53.4 \%)$ & 55 (42.6\%) & $1.54(0.95-2.51)$ & .082 & $0.61(0.31-1.22)$ & .162 \\
\hline Death & $48(36.1 \%)$ & $55(42.6 \%)$ & $0.76(0.46-1.25)$ & .279 & $1.70(0.86-3.33)$ & .125 \\
\hline
\end{tabular}




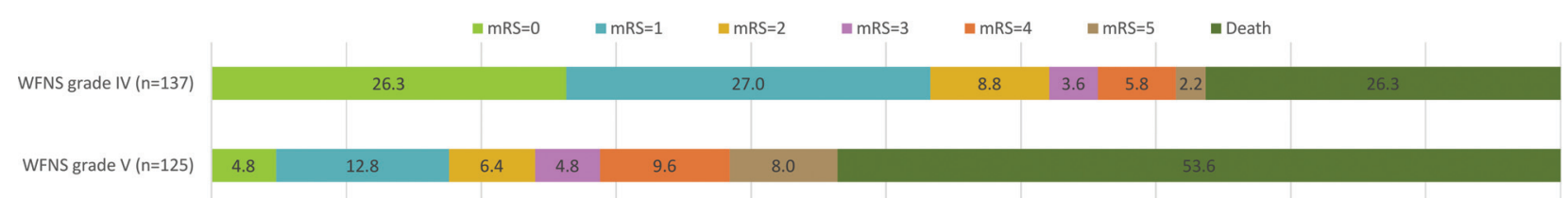

$\begin{array}{lllllllll}\text { A } & 0 \% & 10 \% & 20 \% & 30 \% & 40 \% & 50 \% & 60 \% & 70 \%\end{array}$

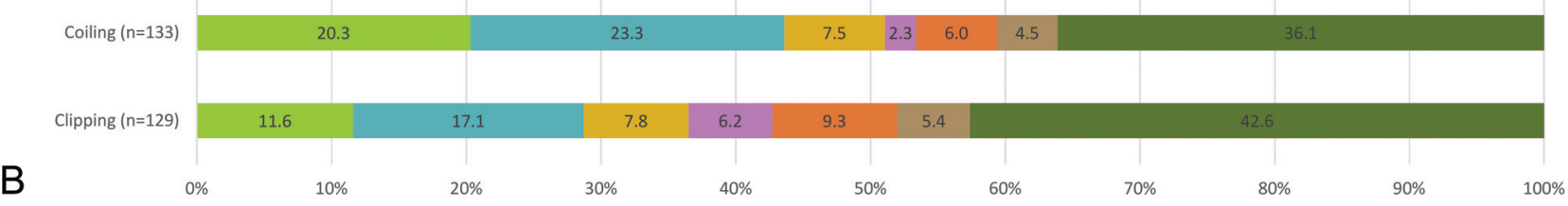

FIG 2. Outcome at 12 months in all patients. $A$, Outcome according to WFNS grade. $B$, Outcome by type of treatment in all patients.

had a better outcome than those treated with clipping at 6 and 12 months. Selected patients with a WFNS grade of IV were treated with coiling and might have a better outcome. After we adjusted for differences in baseline risk factors, these outcomes after coiling were similar to those after clipping. In a prospective data base of poor-grade aneurysms, Mocco et $\mathrm{al}^{2}$ reported that $35(35.7 \%)$ of 98 patients underwent coiling, and there was similar outcome between coiling and clipping. The current results at the 3- and 6-year follow-ups in the Barrow Ruptured Aneurysm Trial also showed that there was no significant difference in outcome between the 2 treatments for ruptured aneurysms. ${ }^{17,18}$ These results suggest that both endovascular coiling and surgical clipping are viable treatment options for poor-grade ruptured aneurysms. Clinicians should make treatment decisions case-by-case, weighing the benefit of different treatments. Furthermore, with improvements in endovascular technology and surgical techniques, aneurysm treatment does not seem to make a big difference in the outcomes of selected patients.

Previously, no study focused on poor-grade aneurysms to determine the effects of coiling and clipping on the postoperative complications. In our study, radiologic hydrocephalus was used to make it more appropriate to assess these complications at all sites. Our results are in line with a meta-analysis showing a significantly higher risk for hydrocephalus after coiling than clipping for ruptured aneurysms. ${ }^{19}$ Our study also showed a trend toward clinical hydrocephalus in the coiling group because open surgery may remove the clot and open cistern and result in less hydrocephalus. ${ }^{19}$ However, Zaidi et $\mathrm{al}^{20}$ reported no difference in hydrocephalus among patients treated by coiling or clipping. There is still a controversy regarding the effect of treatment technique on hydrocephalus, probably because of different diagnostic criteria of hydrocephalus and the time period observed. In addition, all patients had poor neurologic conditions, and systematic vasospasm could not be precisely detected. The long-term complications were also not determined because due to a high mortality in poor-grade patients, it might be difficult to properly compare these complications across time.

To the best of our knowledge, this is the first multicenter prospective report focused on the effects of postoperative complications and clinical outcome between the 2 treatments for poorgrade ruptured aneurysms. However, our study has several limitations. First, it was not a randomized study, and the drawback is that selected patients were included for the exploratory analysis. All patients were treated according to the individual patient and aneurysm characteristics on the basis of multidisciplinary decision-making. Other factors such as family desires, relative treatment risk, and medical expenses may influence the decisions about treatment technique. Therefore, the decision regarding treatment of poor-grade aneurysms is complex and could not be defined in a registry study. Although we have adjusted for baseline imbalance between the 2 treatments, other unmeasured variables outside the analysis may contribute to selection bias. Therefore, no direct causal effect between treatment and complications and clinical outcome could be ascertained. Further studies focused on poor-grade aneurysms are needed to help guide treatment decisions. Second, we did not record laboratory findings and long-term imaging follow-up, and there may be other risk factors for outcome. Nevertheless, we found no difference in long-term outcomes between the 2 treatments by using a relatively large number of poor-grade patients, but the higher risk of radiologic hydrocephalus should be considered after endovascular coiling. These results may also help clinicians assess and treat the postoperative complications after both treatments.

\section{CONCLUSIONS}

Unadjusted comparisons of outcomes showed that patients undergoing coiling had a better outcome than those undergoing clipping. Selected patients who were treated with coiling may have a better outcome. Adjusted analysis showed that the outcomes at 6 and 12 months were similar between the 2 treatments. A clinical trial focused on poor-grade ruptured aneurysms would be necessary to further assess the efficacy of different treatments. The higher risk of radiologic hydrocephalus was found in patients after endovascular coiling for poor-grade ruptured aneurysms. Meanwhile, there was a trend toward clinical hydrocephalus in patients treated with coiling.

\section{ACKNOWLEDGMENTS}

AMPAS Study Investigators: Hongqi Zhang, MD, Xuan Wu Hospital, Capital Medical University; Chuansheng Liang, MD, The First Hospital of China Medical University; Huaizhang Shi, MD, The First Affiliated Hospital of Harbin Medical University; Jing $\mathrm{Xu}, \mathrm{MD}$, The Second Affiliated Hospital, School of Medicine, Zhejiang University; Li Pan, MD, Wuhan General Hospital of Guangzhou Command; Xin Zhang, MD, Nanjing General Hospital of Nanjing Command; Gang Zhu, MD, West South Hospital, 
The Third Military Medical University; Jianping Deng, MD, Tang Du Hospital, The Fourth Military Medical University; and Zhigang Wang, MD, The Second Affiliated Hospital, School of Medicine, Shandong University.

Disclosures: Bing Zhao—RELATED: Grant: Chinese Ministry of Health, ${ }^{*}$ Comments: They were not involved in the data collection and analysis and the writing of the manuscript. Ming Zhong—RELATED: Grant: Chinese Ministry of Health,* Wenzhou Bureau of Science and Technology, ${ }^{*}$ Comments: The funders were not involved in data collection, interpretation, and analysis. *Money paid to the institution.

\section{REFERENCES}

1. Le Roux PD, Elliott JP, Newell DW, et al. Predicting outcome in poor-grade patients with subarachnoid hemorrhage: a retrospective review of 159 aggressively managed cases. J Neurosurg 1996;85: 39-49 CrossRef Medline

2. Mocco J, Ransom ER, Komotar RJ, et al. Preoperative prediction of long-term outcome in poor-grade aneurysmal subarachnoid hemorrhage. Neurosurgery 2006;59:529-38; discussion 529-38 CrossRef Medline

3. Huang AP, Arora S, Wintermark M, et al. Perfusion computed tomographic imaging and surgical selection with patients after poorgrade aneurysmal subarachnoid hemorrhage. Neurosurgery 2010; 67:964-74; discussion 975 CrossRef Medline

4. Sandström N, Yan B, Dowling R, et al. Comparison of microsurgery and endovascular treatment on clinical outcome following poorgrade subarachnoid hemorrhage. J Clin Neurosci 2013;20:1213-18 CrossRef Medline

5. Pereira AR, Sanchez-Peña P, Biondi A, et al. Predictors of 1-year outcome after coiling for poor-grade subarachnoid aneurysmal hemorrhage. Neurocrit Care 2007;7:18-26 CrossRef Medline

6. Mitra D, Gregson B, Jayakrishnan V, et al. Treatment of poor-grade subarachnoid hemorrhage trial. AJNR Am J Neuroradiol 2015;36: 116-20 CrossRef Medline

7. Molyneux AJ, Kerr RS, Yu LM, et al; International Subarachnoid Aneurysm Trial (ISAT) Collaborative Group. International Subarachnoid Aneurysm Trial (ISAT) of neurosurgical clipping versus endovascular coiling in 2143 patients with ruptured intracranial aneurysms: a randomised comparison of effects on survival, dependency, seizures, rebleeding, subgroups, and aneurysm occlusion. Lancet 2005;366:809-17 CrossRef Medline

8. Molyneux AJ, Birks J, Clarke A, et al. The durability of endovascular coiling versus neurosurgical clipping of ruptured cerebral aneurysms: 18 year follow-up of the UK cohort of the International Subarachnoid Aneurysm Trial (ISAT). Lancet 2015;385:691-97 CrossRef Medline
9. Suzuki S, Jahan R, Duckwiler GR, et al. Contribution of endovascular therapy to the management of poor-grade aneurysmal subarachnoid hemorrhage: clinical and angiographic outcomes. J Neurosurg 2006;105:664-70 CrossRef Medline

10. Taylor CJ, Robertson F, Brealey D, et al. Outcome in poor grade subarachnoid hemorrhage patients treated with acute endovascular coiling of aneurysms and aggressive intensive care. Neurocrit Care 2011;14:341-47 CrossRef Medline

11. Zhao B, Tan X, Yang H, et al. A Multicenter Prospective Study of Poor-Grade Aneurysmal Subarachnoid Hemorrhage (AMPAS): observational registry study. BMC Neurol 2014;14:1471-2377 CrossRef Medline

12. Zhao B, Tan X, Zhao Y, et al. Variation in patient characteristics and outcomes between early and delayed surgery in poor-grade aneurysmal subarachnoid hemorrhage. Neurosurgery 2015 Sep 29. [Epub ahead of print] Medline

13. Zhao B, Cao Y, Tan X, et al. Complications and outcomes after early surgical treatment for poor-grade ruptured intracranial aneurysms: a multicenter retrospective cohort. Int J Surg 2015;23: 57-61 CrossRef Medline

14. Dorai Z, Hynan LS, Kopitnik TA, et al. Factors related to hydrocephalus after aneurysmal subarachnoid hemorrhage. Neurosurgery 2003;52:763-69; discussion 769-71 CrossRef Medline

15. Sheehan JP, Polin RS, Sheehan JM, et al. Factors associated with hydrocephalus after aneurysmal subarachnoid hemorrhage. $\mathrm{Neu}$ rosurgery 1999;45:1120-27; discussion 1127-28 CrossRef Medline

16. Wilson DA, Nakaji P, Albuquerque FC, et al. Time course of recovery following poor-grade SAH: the incidence of delayed improvement and implications for SAH outcome study design. J Neurosurg 2013; 119:606-12 CrossRef Medline

17. Spetzler RF, McDougall CG, Albuquerque FC, et al. The Barrow Ruptured Aneurysm Trial: 3-year results. J Neurosurg 2013;119:146-57 CrossRef Medline

18. Spetzler RF, McDougall CG, Zabramski JM, et al. The Barrow Ruptured Aneurysm Trial: 6-year results. J Neurosurg 2015;123:609-17 CrossRef Medline

19. de Oliveira JG, Beck J, Setzer M, et al. Risk of shunt-dependent hydrocephalus after occlusion of ruptured intracranial aneurysms by surgical clipping or endovascular coiling: a single-institution series and meta-analysis. Neurosurgery 2007;61:924-33; discussion 933-34 CrossRef Medline

20. Zaidi HA, Montoure A, Elhadi A, et al. Long-term functional outcomes and predictors of shunt-dependent hydrocephalus after treatment of ruptured intracranial aneurysms in the BRAT trial: revisiting the clip vs coil debate. Neurosurgery 2015;76:608-13; discussion 613-14; quiz 614 CrossRef Medline 